\title{
Standard Poodle
}

National Cancer Institute

\section{Source}

National Cancer Institute. Standard Poodle. NCI Thesaurus. Code C53860.

The Standard Poodle is a medium to large-sized dog with a height of 15 inches or more and a weight of 45-70 pounds. 